1

2

3

4

5

6

7

8

9

10

11

12

13

14

15

16

17

18

\title{
Proteoglycans exert a significant effect on human meniscal stiffness through ionic effects
}

Mr Fahd Mahmood ${ }^{1,2}$, Mr Jon Clarke ${ }^{2}$, Dr Philip Riches ${ }^{1}$

1. Department of Biomedical Engineering, Wolfson Centre, University of Strathclyde, 16 Richmond Street, Glasgow, G1 1XQ, UK

2. Department of Orthopaedics, Golden Jubilee National Hospital, Agamemnon Street, Clydebank, G81 4DY, UK

Corresponding author: Fahd Mahmood (fahdmahmood@hotmail.com)

\section{Abstract \\ Background}

Proteoglycans contribute to mechanical stiffness in articular cartilage, aiding load transmission. The magnitude of the ionic contribution of proteoglycans to the stiffness of human meniscal tissue has not been established.

\section{Methods}

Thirty-six discs of human meniscal tissue were placed within a custom confined compression chamber and bathed in three solutions of increasing ionic concentration. Following a $0.3 \mathrm{~N}$ preload, at equilibrium, a $10 \%$ ramp compressive strain was followed by a 7200 second hold phase. A nonlinear poroviscoelastic model with strain dependent permeability was fitted to resultant stress relaxation curves. All samples were assayed for proteoglycan content. Model parameters were analysed using multivariate analysis of variance whilst proteoglycan content was compared using a univariate analysis of variance model.

\section{Findings}

A significant difference $(p<0.05)$ was observed in the value of the Young's modulus $(E)$ between samples tested in deionised water compared to those tested in solutions of high ionic concentration. No differences were observed in the zero-strain permeability or the exponential strain dependent stiffening coefficient. Proteoglycan content was not found to differ with solution; but was found to be significantly increased in the middle meniscal region of both menisci.

\section{Interpretation}

Proteoglycans make a significant ionic contribution to mechanical stiffness of the meniscus, increasing it by $58 \%$ in the physiological condition. It is therefore critical that meniscal regeneration strategies attempt to recreate the function of proteoglycans to ensure normal meniscal function.

Keywords: meniscus; proteoglycans; tissue mechanics

Word count (abstract): 228 words

Word count: 3072 words (excluding references)

Declarations of interest: None 


\section{Introduction}

Whilst, historically, the orthopaedic community has been oblivious to the importance of the menisci to the normal functioning of the knee, it is now appreciated that the meniscus serves a number of functions including load transmission [1], aiding congruity of the joint surfaces [2] and in stability of the knee [3], especially in ACL (anterior cruciate ligament) deficient states [4].

It is well established [1] that the structure of the meniscus aids it in transmitting load. The electrolyte content of the meniscus is estimated at $74 \%$ [5], with $80 \%$ of the remaining dry weight being Type 1 collagen [6]. Collagen fibres are oriented circumferentially in the deep layers of the meniscus, parallel to the meniscal border, with radial and axial oriented tie fibres branching from the peripheral border of the meniscus to its inner rim, surrounding the aforementioned circumferential fibres $[1,7]$. The menisci are firmly anchored to the tibial surfaces at their roots. Under load, the firm attachment of the menisci at their roots prevent extrusion and allow generation of circumferential tensile hoop stresses in the circumferential collagen fibres, aiding load distribution [8]. Some superficial fibres are oriented radially, interweaving between the circumferential fibres, providing structural integrity.

As well as collagen, the meniscus is also comprised of fibrochondrocytes and proteoglycans. Proteoglycans are proteins with numerous sulphated glycosaminoglycan side chains carrying a strong negative charge. Aggrecan is the major type of large proteoglycan found within the meniscus [9] and proteoglycans are thought to comprise 2-3\% of the dry weight of the meniscus [6], with the distribution of proteoglycans in the tissue varying in both frontal and coronal planes [10]. These proteins are highly hydrophilic and allow water to be trapped within the tissue, supporting the tissue under compressive load [11]. Whilst aggrecan is also present as one of the dominant proteoglycans in both articular cartilage and the nucleus pulposus of the intervertebral disc, the proportion of proteoglycans in both these tissue is close to an order of magnitude higher than that observed in the meniscus [12].

In articular cartilage, proteoglycans are understood to play a major role in maintaining the compressive stiffness of the tissue, with digestion of proteoglycans resulting in a marked reduction in its compressive modulus [13] and correlation evident between proteoglycan content and this modulus. In cartilage, the strong negative charge of proteoglycan molecules exerts a Donnan osmotic pressure: as the negatively charged moieties attached to the proteoglycans are fixed in the meniscal ultrastructure, charge is distributed unevenly across the cartilage membrane, leading to development of an electrical potential (the Donnan potential) across the cellular boundary. This, in turn leads to generation of an osmotic pressure and inflow water into the cartilage, ultimately allowing fluid to be absorbed into the cartilage to aid load resistance. Study of articular cartilage to investigate the contribution of the ionic effect of proteoglycans to stiffness of the tissue suggests that $62 \%$ of the compressive modulus at equilibrium is attributable to such effects [14]. Similar work [15] in the nucleus pulposus of the intervertebral disc suggests that $70 \%$ of the stress response is attributable to ionic effects mediated by proteoglycans, despite the population of proteoglycans in the nucleus pulposus being composed of short length monomers as opposed to the larger chain polymeric molecules observed in other tissues [16].

There has been limited exploration of the role of proteoglycans in maintaining mechanical stiffness of the meniscus to date. Evaluation of cervine meniscal stiffness using microindentation techniques following use of hyaluronidase to digest glycosaminoglycans showed a reduction of $15 \%$ in creep stiffness [17]. Also, a recent study [18] suggested that proteoglycans within the meniscus exert a significant effect on mechanical stiffness via ionic effects, although the number of samples and the 
use of bovine meniscus limits how far these results can be extrapolated. Therefore, this study aims to quantify the contribution that proteoglycans make to the stiffness of the human meniscus.

\section{Methods}

Following ethical approval, 12 paired, fresh frozen human menisci were obtained from a tissue repository. Donors were less than 65 years of age, with no history of knee surgery, knee osteoarthritis or significant knee injury. Samples were defrosted on the morning of experimentation.

A hollow punch was used to obtain $36,5 \mathrm{~mm}$ diameter sections of meniscal tissue from either the anterior, middle or posterior regions of each meniscus (Figure 1). All sampling was conducted from the periphery of the meniscus to allow a cylindrical sample of sufficient dimensions to be obtained.

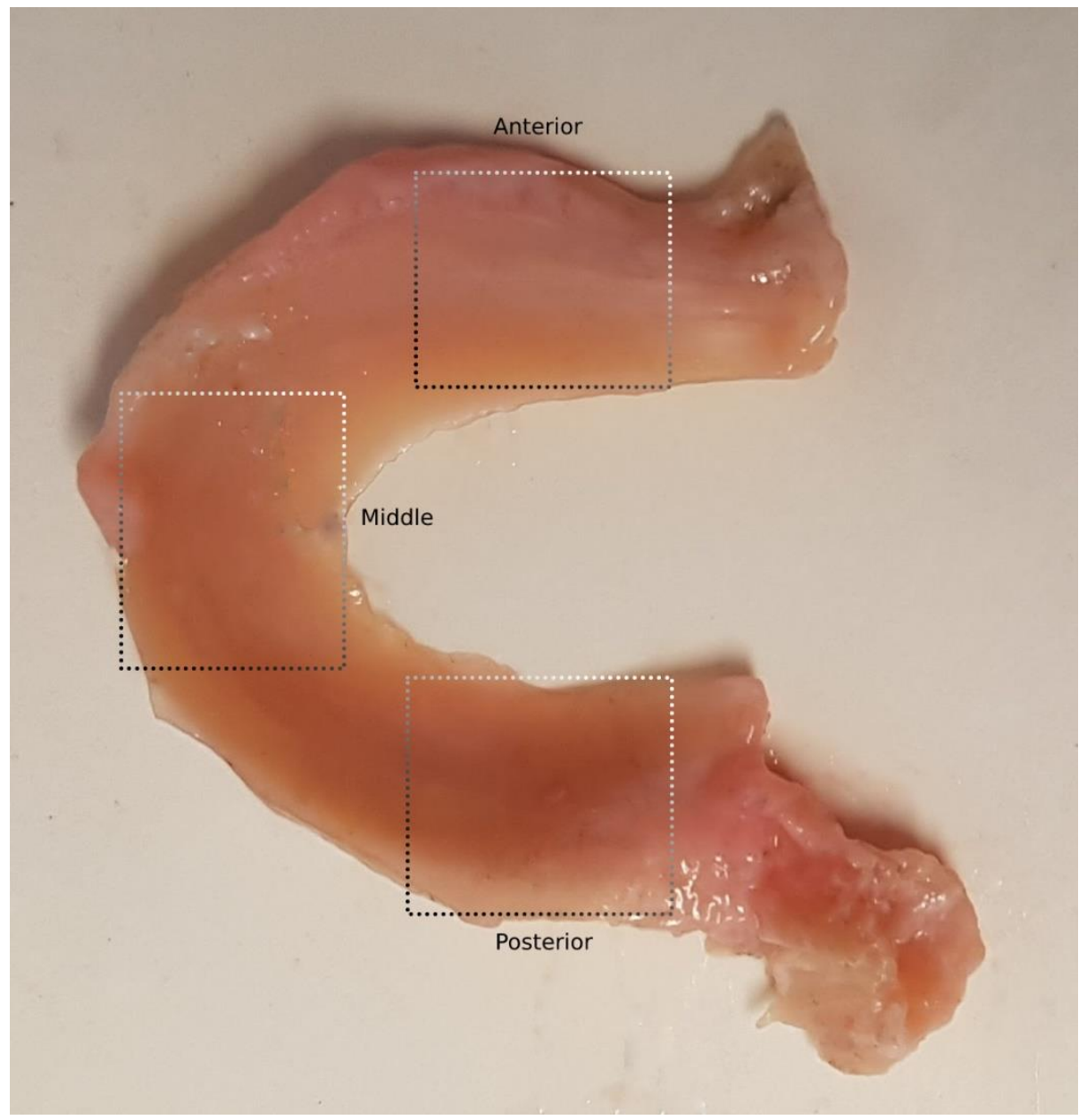

Figure 1 - Superior view of a right knee lateral meniscus illustrating meniscal sample locations

Due to the topography of the menisci, the superior and inferior surfaces of the sections were not parallel and therefore a custom-made device, which held two microtome blades precisely $2 \mathrm{~mm}$ apart, was used to obtain a cylindrical sample from the centre of each section, such that both contact surfaces were removed. Sample thickness was determined using a micrometer screw gauge.

A bespoke confined-compression chamber, with an inner-diameter of $5 \mathrm{~mm}$ and a bottom latticework of $400 \mu \mathrm{m}$ square pores, was 3D printed. A hollow indenter, which also had a permeable lattice at its inferior aspect fitted the chamber, ensuring a small side-clearance. The indenter gripped by a BOSE 3100 materials testing machine fitted with a $22 \mathrm{~N}$ load cell (Figure 2). Similar techniques have 

articular cartilage [20].

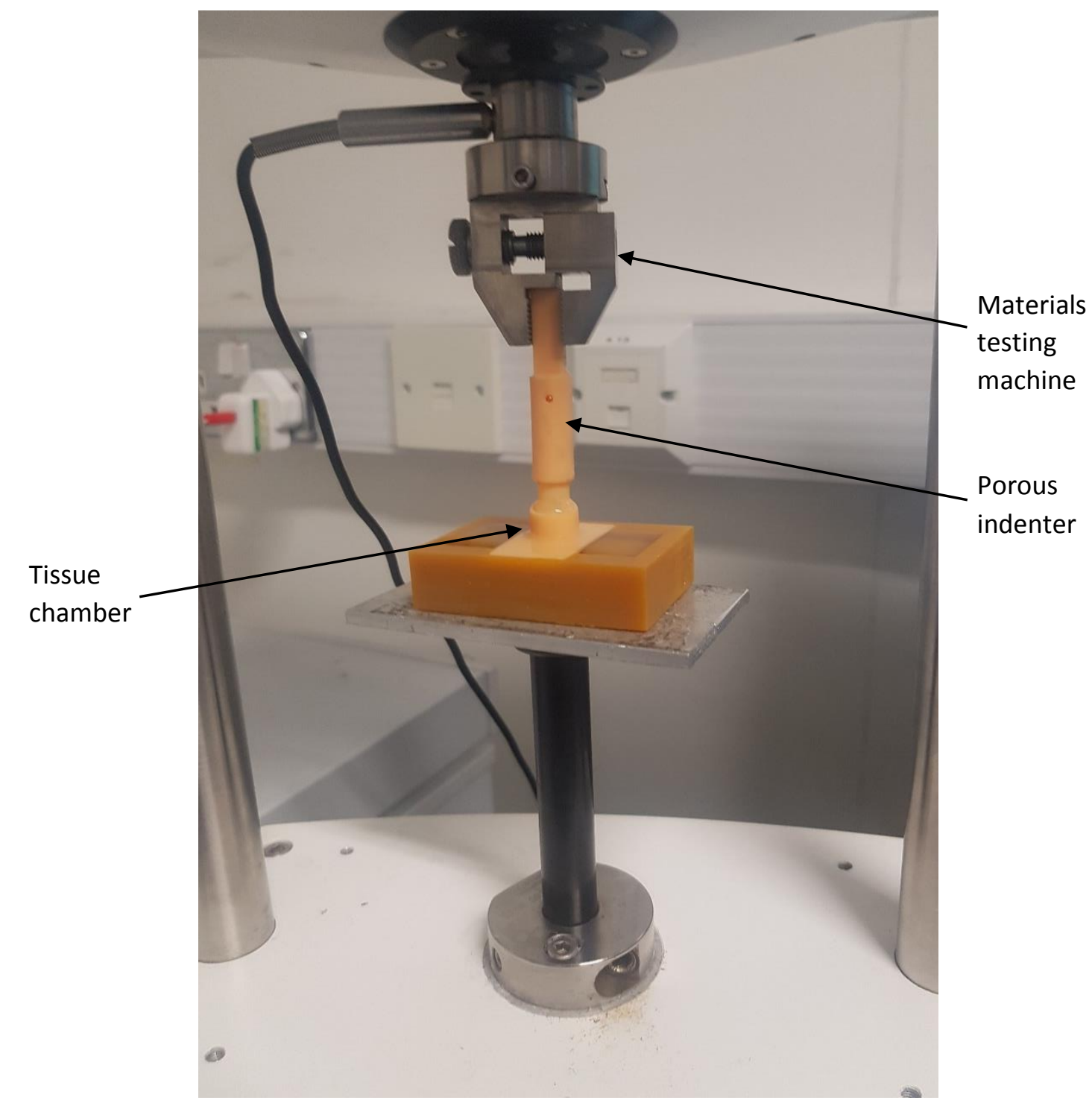

Figure 2 - Confined compression apparatus (bathing chamber removed for clarity)

Each of the 36 samples were placed within the compression chamber and bathed in in one of three solutions, such that the fluid permeated the tissue, the confining chamber and the indenter. As in previous studies $[9,14]$, deionised water was used to negate the osmotic effects of mobile ions within the tissue; $0.14 \mathrm{M}$ PBS (phosphate buffered saline) was used to mimic a physiological environment and 3M PBS negate all ionic effects. Four samples from the anterior, middle and posterior regions were tested in each solution, such that specimens from different anatomical regions were distributed evenly across each experimental group.

The meniscal surface was identified by lowering the indenter until a load of $0.3 \mathrm{~N}$ was registered. The sample was then allowed to equilibrate, holding the displacement of the indenter constant. The time to equilibrium was determined by holding the displacement until the force measured did not vary by $>2 \%$ over a 30 minute period. Time to reach equilibrium was 2 hours for samples tested in $0.14 \mathrm{M}$ PBS and 3M PBS, whilst samples tested in deionised water were left to equilibrate for 15 hours. A 

7200 s. Each sample was tested once.

A finite element model was constructed using FEBio software using a non-linear, large strain, biphasic, poroviscoelastic model with strain dependent permeability. This model assumes the tissue as having two phases. The viscoelastic solid phase was defined by an elastic stress given by (Homes and Mow, 1990)

$$
\sigma_{e}=\frac{1}{2} H_{M}\left(\frac{\lambda^{2}-1}{\lambda^{2 \beta+1}}\right) e^{\beta\left(\lambda^{2}-1\right)}
$$

where $\lambda$ is the stretch $(\lambda=1+\varepsilon) ; H_{M}$ the gradient of the stress-strain curve at $\lambda=1$; and $\beta$ a stiffening coefficient associated with the sensitivity of $\sigma_{e}$ to large strain. The solid phase's relaxation function $G(t)$ was described by

$$
G(t)=1+G_{1} e^{-\frac{t}{\tau_{1}}}
$$

The fluid phase was an incompressible Newtonian fluid. Time-dependent behaviour is associated with the resistance to the flow of fluid through the solid via Darcy's law and characterised by the hydraulic permeability (permeability divided by the viscosity of the permeating fluid). A variation on the Holmes-Mow model of strain-dependent permeability was used, i.e.

where the hydraulic permeability is dependent on the stretch and $\phi_{0}$ is the zero-strain porosity. The zero-strain permeability, $k_{0}$, and the exponential strain dependent coefficient, $M$, were restricted from becoming negative, whilst the power law exponent, $\alpha$, was held at zero, reducing the above equation to

$$
k(\lambda)=k_{0}\left(\frac{\lambda-\phi_{0}}{1-\phi_{0}}\right)^{\alpha} e^{\left[M\left(\lambda^{2}-1\right) / 2\right]}
$$

Such a model has been used in the literature to describe both articular cartilage and meniscus $[19,21]$. The model consisted of 404 nodes, with 100 elements. A convergence study was conducted to investigate an appropriate model size, this suggested a percentage error of $<0.1 \%$ for a model with 404 nodes compared to one with $\sim 1000$ nodes. Hence a 404-node model was chosen to allow an acceptable compromise between accuracy and computational calculation efficiency. Boundary conditions were set appropriate to confined compression, and the Poisson's ratio was set to zero, so that for small strains, $H_{M} \cong E$, the Young's modulus.

Model parameters were determined by fitting the experimental force on the sample in the relaxation phase to the calculated force in the FE model using the fminsearch function in Matlab (Mathworks, Massachusetts, USA) and $\mathrm{E}, k_{0}, M, \beta, G_{1}$ and $\tau_{1}$ as fitting parameters. After each iteration, matlab amended the model and called the FEBio solver. The goodness of fit in the stress relaxation fit was assessed using a coefficient of determination as described by Soltz and Ateshian [19]. Converged best fit parameters were compared using multivariate ANOVA with Bonferroni correction for multiple comparisons, with meniscal side, meniscal region and bath osmolarity as fixed factors and with significance set at $p \leq 0.05$. Once tested, samples were immediately re-frozen.

Proteoglycan content in each sample was determined by a proprietary assay (Biocolour Ltd, County Antrim, UK). Prior to applying the assay, all samples were washed with deionised water 10 times to remove any excess salt, as the results of the assay could be skewed by presence of excessive salt. 
A papain extraction reagent was prepared by adding $800 \mathrm{mg}$ sodium acetate, $400 \mathrm{mg}$ EDTA and $40 \mathrm{mg}$ cysteine hydrochloride to $100 \mathrm{ml}$ of a $0.2 \mathrm{M}$ sodium phosphate buffer. The $\mathrm{pH}$ of this solution was corrected to 6.4 and 250 microlitres of a papain crystallised suspension was added. Each sample was then cut in half using a scalpel and its wet weight was recorded - the assay required a wet weight of 20-50mg. Individual samples were them placed in labelled microcentrifuge tubes with $1 \mathrm{ml}$ of the papain extraction reagent. All samples were placed in a warm water bath $\left(65^{\circ} \mathrm{C}\right)$ and set to shake slowly. Samples were left overnight to digest, then centrifuged at $10000 \mathrm{~g}$ for 10 minutes

$50 \mu l$ of the supernatant of each test sample was added to individual microcentrifuge tubes and made up to $100 \mu \mathrm{l}$ using the previously prepared papain extraction reagent. Tubes containing $1,2,3,4,5 \mu \mathrm{g}$ of the assay reference standard were also prepared and made up to $100 \mu \mathrm{l}$ in a similar fashion. A millilitre of the assay dye reagent was then added to each sample.

All samples were placed on a mechanical shaker for 30 minutes, during which time a precipitant was observed to form, a further 10 minutes of centrifugation was undertaken at $12000 \mathrm{~g}$. The supernatant from each tube was then carefully removed and $0.5 \mathrm{ml}$ of the dye dissociation reagent added. A vortex mixer was used to allow the bound dye to dissolve into solution and $200 \mu \mathrm{l}$ of each sample was transferred to a 96 microwell plate. A microplate reader was used to measure absorbance at $656 \mathrm{~nm}$.

The luminescence values for the assay reference standard solutions were used to create a standard curve. A best fit line was applied to this curve using Microsoft Excel (Microsoft, Redmond, Washington, United States) - the equation for this line was then used to calculate proteoglycan content for individual samples. Proteoglycan content was compared between samples using univariate ANOVA, with significance set at $p \leq 0.05$. Proteoglycan content was considered as the sole dependent variable, with the solution tested, the meniscus tested, and the region of the sample considered as fixed factors.

\section{Results}

Thirty six samples were obtained from 12 menisci, three from each meniscus. Mean sample thickness was 1.99 (SD 0.04) mm.

Following best fitting, the viscoelastic coefficient, $G_{1}$, and relaxation time, $\tau_{1}$, were close to zero in all samples, hence finite element modelling was conducted twice for all samples: with and without $G_{1}=0$. No difference was observed in the other material parameters between these two conditions, and thus it was assumed that no viscoelastic behaviour of the solid phase occurred. If one assumes that solid phase viscoelasticity is related to the viscoelastic stress relaxation of collagen fibre tension in the matrix, compression and buckling of the fibrous solid phase could be considered unlikely to elicit a viscoelastic effect. $G_{1}$ was therefore prescribed to be zero and is not presented or discussed further.

Table 1 shows the derived values for the mechanical parameters of the tissue in each solution. 
Parameter mean values (95\% confidence interval)

\begin{tabular}{|c|c|c|c|c|}
\hline \multicolumn{5}{|c|}{ Parameter mean values (95\% confidence interval) } \\
\hline Solution & $\begin{array}{c}\text { E } \\
\text { (Young's } \\
\text { Modulus) (MPa) }\end{array}$ & $\begin{array}{c}\mathbf{k}_{0} \\
\text { (zero strain } \\
\text { dependent } \\
\text { permeability) } \\
\left(\times 10^{-16} \mathrm{~m}^{4} / \mathrm{Ns}\right)\end{array}$ & $\begin{array}{c}\text { M } \\
\text { (exponential } \\
\text { strain dependent } \\
\text { coefficient) }\end{array}$ & $\begin{array}{c}\beta \\
\text { (exponential } \\
\text { stiffening } \\
\text { coefficient) }\end{array}$ \\
\hline Deionised water & $1.15(0.94-1.35)^{*}$ & $0.08(0.00-0.22)$ & $0.01(0.10-0.12)$ & $0.23(0.18-0.28)$ \\
\hline $0.14 \mathrm{M}$ PBS & $0.68(0.48-0.89)$ & $0.24(0.10-0.38)$ & $0.01(0.10-0.12)$ & $0.23(0.18-0.28)$ \\
\hline 3M PBS & $0.43(0.22-0.63)$ & $0.18(0.04-0.32)$ & $0.01(0.10-0.12)$ & $0.21(0.17-0.26)$ \\
\hline \multicolumn{5}{|c|}{ * $\mathrm{p}<0.05$ compared to $0.14 \mathrm{M} / 3 \mathrm{M}$ PBS. } \\
\hline
\end{tabular}

Table 1 - Parameter mean values

A significant difference was observed in the value of the Young's modulus between samples tested in deionised water compared to those tested in either 0.14M $(p=0.01)$ or 3M PBS $(p<0.01)$. No significant differences were observed in either the value of the zero-strain hydraulic permeability or the exponential strain dependent, or the exponential stiffening coefficient between solutions. Furthermore, no significant differences were observed in the value of any of the mechanical parameters when comparing meniscal side or meniscal region. The mean $R^{2}$ values, assessing goodness of fit, were $0.83,0.75$ and 0.76 in deionised water, $0.14 \mathrm{M}$ PBS and $3 \mathrm{M}$ PBS respectively, with an overall mean $R^{2}$ of $0.78+/-0.11$ (s.d.).

Proteoglycan content was not found to differ significantly with solution: Table 2 illustrates the mean concentration of proteoglycans across solutions. However, proteoglycan content was found to be increased in the middle region of each meniscus ( $p=0.043$ vs anterior samples, $p=0.036$ vs posterior samples).

\begin{tabular}{|c|c|}
\hline Region & $\begin{array}{c}\text { Proteoglycan content }(\boldsymbol{\mu g} / \mathbf{g} \\
\text { of tissue) [95\% confidence } \\
\text { interval] }\end{array}$ \\
\hline Deionised water & $196.05[159.37-232.73]$ \\
\hline $0.14 \mathrm{M}$ PBS & $151.28[114.60-187.96]$ \\
\hline 3M PBS & $148.67[139.46-212.82]$ \\
\hline
\end{tabular}

Table 2 - Proteoglycan content

\section{Discussion}

Proteoglycans make a significant ionic contribution to mechanical stiffness of the human meniscus, in the non-diseased state, increasing the meniscal stiffness by $58 \%$ in the physiological condition compared to the $3 \mathrm{M}$ state. Despite the marked difference in the concentration of proteoglycans in meniscus compared to articular cartilage or intervertebral disc, the magnitude of this contribution is 
not too dissimilar to that described for these tissues. Hence, proteoglycans are integral to meniscal function and any efforts to repair or re-constitute the tissue should account for their function.

The ramifications of this finding could be clinically significant. To our knowledge, there have been no studies to date exploring the constitution of either healed meniscal tissue following primary repair or allograft tissue following transplantation with respect to its proteoglycan content and whether this is similar to that observed in the native tissue. An animal study has suggested that supplementation of meniscal repairs with hepatocyte growth factor/ platelet derived growth factor results in increased proteoglycan staining compared to that observed in menisci repaired without growth factors [22]. Furthermore, evaluation of biopsies of the Actifit meniscal scaffold have shown proteoglycans in only a proportion of samples, albeit in a small patient population [23]. Interestingly, seeding such scaffolds with biologically active constituents such as growth factors or stem cells may provide a means through which to encourage healing and/or proteoglycan reconstitution, an option which is being explored [24]. If meniscal defects heal without the presence of proteoglycans within the tissue, the resultant tissue is likely to be less stiff than native meniscus and may therefore be inferior in its ability to transmit load, leading to abnormal stresses on adjacent articular cartilage.

The tissue is markedly stiffer in deionised water than in either PBS solution. This can be explained by the fact that, in the absence of mobile ions, the stiffness of the tissue is significantly augmented by the osmotic pressure generated by the difference in fixed charge density between the internal and external environment. Circulating mobile ions internally and externally in the physiological condition reduce the electrostatic disparity, whilst the hypertonic condition is designed to nullify the electrostatic effects completely. Although it did not reach significance, the permeability of the tissue was also lowest in deionised water. Transiently, an isotonic permeate retains both mobile and fixed ion gradients which enhance fluid flow within tissue for a given mechanical fluid pressure [25]. Thus, the lower permeability seen in hypertonic and hypotonic solutions are not mechanical low permeability pe se, rather reduced, apparent permeabilities due to the loss of these gradients. Nonetheless, whilst a biphasic model can identify and partition the overall effects of the osmolarity of the permeate, a triphasic [26] or quadriphasic model [27] is required to truly and fully describe the physics of the experiment.

242 Neither the exponential stiffening nor strain dependent coefficients differed between solutions.

243 These variables seem unaffected by ionic changes within the solution; however, their similarity adds credence to the reliability of the experimental technique.

Whilst we fitted a nonlinear poroviscoelastic model to the data, $G_{1}$ and $\tau_{1}$ were found to be zero, indicating one of two things: either the collagen network did not exhibit stress relaxation, or that the curve fitting algorithm iterated towards a local best-fit solution in which $\mathrm{G}_{1}$ and $\tau_{1}$ were equal to zero. In confined compression, the likelihood of stress relaxation within collagen fibres themselves is difficult to argue and thus we suggest this finding infers this. Regardless, this model does not consider ionic effects and future work may explore the appropriateness of a triphasic model, described by Lai et al [26], in predicting the behaviour of meniscal tissue. Similar to a poroviscoelastic model, such an approach is challenging due to the likelihood of obtaining multiple 'false positive' solutions [28] due to the multitude of parameters being fitted. confounder for our experiments. Nevertheless, we found that proteoglycan content is increased in the middle region of each meniscus. Interestingly, this did not lead to an increase in stiffness of this region compared to anterior or posterior samples, suggesting that the maximal ionic contribution of 
proteoglycans to meniscal stiffness is either not concentration dependent, or limited by other factors.

260 Our study has a number of strengths. We have tested a large number of samples, using a common technique. By testing samples at equilibrium, we have excluded any potential effects of swelling, which can be significant in meniscal tissue [29]. Potential weaknesses of our work are the use of frozen tissue, although fresh frozen tissue is commonly used in biomechanical testing. Nonetheless, examination of fresh tissue may show different results. We were unaware of the post-mortem time region, but rather chose to test equal numbers of samples from each region, as this would have required a large number of menisci. Previous work [30] has suggested that mechanical behaviour of meniscal tissue is altered dependent on the region it is derived from, our work did not show such a difference, although we did have small numbers of samples from each region. In any case, testing equal numbers of samples from each meniscal region allowed for any effect of such differences to remain equal between solutions. As well as this, our samples were all derived from the periphery of the meniscus - proteoglycan concentration has been shown to be highest in the inner zones of the meniscus [31]. Our sampling technique did not allow us to differentiate between the vascular zones of the meniscus. We also did not use protease inhibitors to prevent specimen degradation.

A mean R2 value of $0.78+/-0.11$ suggest good, but not excellent fits, with the model struggling to fit at early times in the hold phase. The equilibrium force determined Young's modulus and thus the permeability value controlled not only the initial peak compressive force, but also the rate of stress relaxation observed. In decreasing $\mathrm{k} 0$ one obtains a higher negative peak force, but slower mechanics, whilst increasing $\mathrm{k} 0$, decreased peak force and increased the rate of relaxation. It was difficult for the model to accurately capture both the peak load and the rate of relaxation, and in the majority of cases a compromise $\mathrm{k} 0$ was converged to. Additional model parameters (beta, $\mathrm{G} 1$ tau1 etc.) were not able to further improve the fit. Thus, the constitutive behaviour of the model and its appropriateness for meniscal tissue warrants additional research.

Whilst the ionic concentrations sampled here are supra-physiological, this works highlights that proteoglycans significantly contribute to meniscal stiffness at physiological ion concentrations via ionic effects. Whilst no prosthetic replacement for meniscal tissue currently exists, there has been interest in the use of meniscal scaffolds such as the Actifit (Orteq Ltd, Wimbledon, London, United Kingdom) and Collagen Meniscus Implant (Ivy Sports Medicine GmbH, Grafelfing, Germany). Tissue ingrowth following the use of such implants has been found to be composed of collagen [32,33], however, there has been no consideration of whether proteoglycans are adequately restored. This work highlights the importance of restoring normal proteoglycan function is such meniscal preservation strategies.

\section{Conclusion}

Proteoglycans make a significant contribution via ionic effects to the stiffness of the human meniscus, increasing the stiffness by $58 \%$. These data suggest that meniscal preservation strategies should take this contribution into account and seek to reconstitute proteoglycans within the tissue, allowing repaired tissue to mimic the properties of the native tissue. Research Society and the Golden Jubilee Research Foundation for this work. 
303 [1] Fithian DC, Kelly MA, Mow VC. Material properties and structure-function relationships in the menisci. Clin Orthop Relat Res 1990;252:19-31.

[2] Renström P, Johnson RJ. Anatomy and biomechanics of the menisci. Clin Sports Med 1990;9:523-38.

[3] Arno S, Hadley S, Campbell KA, Bell CP, Hall M, Beltran LS, et al. The Effect of Arthroscopic Partial Medial Meniscectomy on Tibiofemoral Stability. Am J Sports Med 2013;41:73-9. doi:10.1177/0363546512464482.

[4] MusahI V, Citak M, O'Loughlin PF, Choi D, Bedi A, Pearle AD. The effect of medial versus lateral meniscectomy on the stability of the anterior cruciate ligament-deficient knee. Am J Sports Med 2010;38:1591-7. doi:10.1177/0363546510364402.

[5] Sladojević I, Krivokuća Z, Gajanin V, Manojlović S. Expression of Collagen Type 1 in unaltered and osteoarthritic menisci of the knee joint. Med Pregl 2016;69:16-23.

[6] Herwig J, Egner E, Buddecke E. Chemical changes of human knee joint menisci in various stages of degeneration. Ann Rheum Dis 1984;43:635-40. doi:10.1136/ard.43.4.635.

[7] Andrews SHJ, Rattner JB, Abusara Z, Adesida A, Shrive NG, Ronsky JL. Tie-fibre structure and organization in the knee menisci. J Anat 2014;224:531-7. doi:10.1111/joa.12170.

[8] McDermott ID, Masouros SD, Amis AA. Biomechanics of the menisci of the knee. Curr Orthop 2008;22:193-201. doi:10.1016/j.cuor.2008.04.005.

[9] Fischenich KM, Coatney GA, Haverkamp JH, Button KD, DeCamp C, Haut RC, et al. Evaluation of meniscal mechanics and proteoglycan content in a modified anterior cruciate ligament transection model. J Biomech Eng 2014;136:0710011. doi:10.1115/1.4027468.

[10] Danso EK, Oinas JMT, Saarakkala S, Mikkonen S, Töyräs J, Korhonen RK. Structure-function relationships of human meniscus. J Mech Behav Biomed Mater 2017;67:51-60. doi:10.1016/j.jmbbm.2016.12.002.

[11] Makris EA, Hadidi P, Athanasiou KA. The knee meniscus: structure-function, pathophysiology, current repair techniques, and prospects for regeneration. Biomaterials 2011;32:7411-31. doi:10.1016/j.biomaterials.2011.06.037.

[12] Chen S, Fu P, Wu H, Pei M. Meniscus, articular cartilage and nucleus pulposus: a comparative review of cartilage-like tissues in anatomy, development and function. Cell Tissue Res 2017;370:53-70. doi:10.1007/s00441-017-2613-0.

[13] Korhonen RK, Laasanen MS, Töyräs J, Lappalainen R, Helminen HJ, Jurvelin JS. Fibril reinforced poroelastic model predicts specifically mechanical behavior of normal, proteoglycan depleted and collagen degraded articular cartilage. J Biomech 2003;36:1373-9.

[14] Canal Guterl C, Hung CT, Ateshian GA. Electrostatic and non-electrostatic contributions of proteoglycans to the compressive equilibrium modulus of bovine articular cartilage. J Biomech 2010;43:1343-50. doi:10.1016/j.jbiomech.2010.01.021.

[15] Heneghan P, Riches PE. The strain-dependent osmotic pressure and stiffness of the bovine nucleus pulposus apportioned into ionic and non-ionic contributors. J Biomech 2008;41:2411-6. doi:10.1016/j.jbiomech.2008.05.025.

[16] Buckwalter JA, Smith KC, Kazarien LE, Rosenberg LC, Ungar R. Articular cartilage and intervertebral disc proteoglycans differ in structure: An electron microscopic study. J Orthop 
[17] Michalek AJ, Kuxhaus L, Jaremczuk D, Zaino NL. Proteoglycans contribute locally to swelling, but globally to compressive mechanics, in intact cervine medial meniscus. J Biomech 2018;74:86-91. doi:10.1016/J.JBIOMECH.2018.04.023.

[18] Mahmood F, Clarke J, Riches P. The ionic contribution of proteoglycans to mechanical stiffness of the meniscus. Med Eng Phys 2019;64:23-7. doi:10.1016/J.MEDENGPHY.2018.12.010.

[19] Martin Seitz A, Galbusera F, Krais C, Ignatius A, Dürselen L. Stress-relaxation response of human menisci under confined compression conditions. J Mech Behav Biomed Mater 2013;26:68-80. doi:10.1016/j.jmbbm.2013.05.027.

[20] Korhonen R., Laasanen M., Töyräs J, Rieppo J, Hirvonen J, Helminen H., et al. Comparison of the equilibrium response of articular cartilage in unconfined compression, confined compression and indentation. J Biomech 2002;35:903-9. doi:10.1016/S0021-9290(02)000520 .

[21] Ateshian GA, Warden WH, Kim JJ, Grelsamer RP, Mow VC. Finite deformation biphasic material properties of bovine articular cartilage from confined compression experiments. J Biomech 1997;30:1157-64.

[22] Bhargava MM, Hidaka C, Hannafin JA, Doty S, Warren RF. Effects of hepatocyte growth factor and platelet-derived growth factors on the repair of meniscal defects in vitro. Vitr Cell Dev Biol - Anim 2005;41:305. doi:10.1290/0503018.1.

[23] Baynat C, Andro C, Vincent JP, Schiele P, Buisson P, Dubrana F, et al. Actifit synthetic meniscal substitute: experience with 18 patients in Brest, France. Orthop Traumatol Surg Res 2014;100:S385-9. doi:10.1016/j.otsr.2014.09.007.

[24] Whitehouse MR, Howells NR, Parry MC, Austin E, Kafienah W, Brady K, et al. Repair of Torn Avascular Meniscal Cartilage Using Undifferentiated Autologous Mesenchymal Stem Cells: From In Vitro Optimization to a First-in-Human Study. Stem Cells Transl Med 2017;6:1237-48. doi:10.1002/sctm.16-0199.

[25] Farrell MD, Riches PE. Ionic osmotic effects increase fluid flow during permeation tests. J Mech Med Biol 2012;12:1250063. doi:10.1142/S0219519412004995.

[26] Lai WM, Hou JS, Mow VC. A triphasic theory for the swelling and deformation behaviors of articular cartilage. J Biomech Eng 1991;113:245-58.

[27] Huyghe JM, Janssen J. Quadriphasic mechanics of swelling incompressible porous media. Int J Eng Sci 1997;35:793-802. doi:10.1016/S0020-7225(96)00119-X.

[28] Riches PE. Sensitivity analysis of permeability parameters of bovine nucleus pulposus obtained through inverse fitting of the nonlinear biphasic equation: effect of sampling strategy. Comput Methods Biomech Biomed Engin 2012;15:29-36. doi:10.1080/10255842.2010.544301.

[29] Andrews SHJ, Rattner JB, Shrive NG, Ronsky JL. Swelling significantly affects the material properties of the menisci in compression. J Biomech 2015;48:1485-9. doi:10.1016/j.jbiomech.2015.02.001.

[30] Chia HN, Hull ML. Compressive moduli of the human medial meniscus in the axial and radial directions at equilibrium and at a physiological strain rate. J Orthop Res 2008;26:951-6. doi:10.1002/jor.20573. 
[31] Nakano T, Dodd CM, Scott PG. Glycosaminoglycans and proteoglycans from different zones of the porcine knee meniscus. J Orthop Res 1997;15:213-20. doi:10.1002/jor.1100150209.

[32] Heijkants RGJC, van Calck R V, De Groot JH, Pennings AJ, Schouten AJ, van Tienen TG, et al. Design, synthesis and properties of a degradable polyurethane scaffold for meniscus regeneration. J Mater Sci Mater Med 2004;15:423-7.

[33] Reguzzoni M, Manelli A, Ronga M, Raspanti M, Grassi FA. Histology and ultrastructure of a tissue-engineered collagen meniscus before and after implantation. J Biomed Mater Res $B$ Appl Biomater 2005;74:808-16. doi:10.1002/jbm.b.30314. 


\section{Declaration of interests}

$\bigotimes$ The authors declare that they have no known competing financial interests or personal relationships that could have appeared to influence the work reported in this paper.

$\square$ The authors declare the following financial interests/personal relationships which may be considered as potential competing interests:

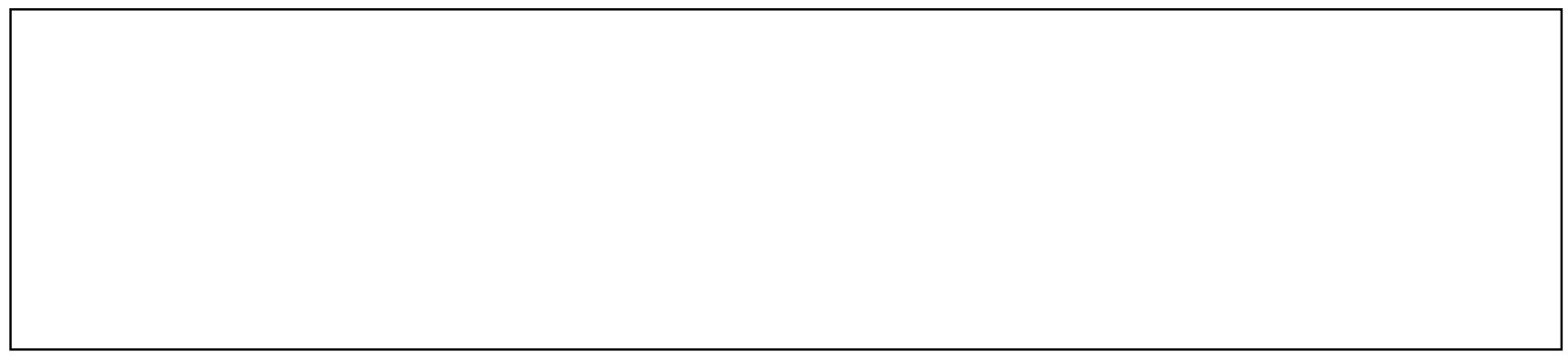

